\title{
Electromagnetic and transient shielding effectiveness for near-field
} sources

\author{
C. Möller and L. Klinkenbusch \\ Christian-Albrechts-Universität zu Kiel, Computational Electromagnetics Group, Kaiserstr. 2, 24143 Kiel, Germany
}

\begin{abstract}
The contribution deals with an investigation of the recently proposed definitions for the electromagnetic and transient shielding effectiveness (SE) in the case of an electric-dipole near-field source. To this end, new factors are introduced which depend on the distance between the dipole source and the measurement point inside the shield and which are valid for perpendicularly (with respect to the distance vector) polarized dipoles. Numerical results support and confirm the theoretical derivations.
\end{abstract}

\section{Introduction}

Shielding against electric, magnetic, and electromagnetic fields is one of the basic steps to ensure the Electromagnetic Compatibility (EMC) of a device or system. The Shielding Effectiveness (SE) is a measure to quantitatively compare the shielding ability of different comparable shields. The common definitions of the electric and magnetic SE are based on the values of either the electric or the magnetic field intensity within an empty shielded domain (Kaden, 1959). Generally, these definitions are suitable and have been proven to be practicable at low frequencies. For higher frequencies and transient incident fields Klinkenbusch (2005) introduced an electromagnetic SE and a transient SE, respectively, which are intended to better characterize the shielding ability for enclosures with dimensions in the resonance regime. The latter definitions have been derived for the case of an incident plane wave, while the present contribution deals with an extension, that is, we derive analog definitions for the case that the source of the electromagnetic field is in the near field of the shield. To this end, we exemplarily investigate that the source is given by an electric (Hertzian) dipole. By introducing simple frequency-dependent factors, we are able to analytically find expressions which relate the electromagnetic and the transient SE to the relatively easily measurable phasors of the electric and magnetic field intensities, in case

Correspondence to: C. Möller

(cm@tf.uni-kiel.de) that the Hertzian dipole source is located in the near field of the shield and polarized perpendicularly with respect to the axis between source and measurement points.

\section{Definitions of the Shielding Effectiveness}

\subsection{Electric and magnetic Shielding Effectiveness (SE)}

The commonly used electric and magnetic SE at an arbitrary point $q$ are defined as (Kaden, 1959):

$\left.\mathrm{SE}_{e}\right|_{q}=20 \log _{10} \frac{\left|\boldsymbol{E}^{u s}\right|_{q}}{\left|\boldsymbol{E}^{s}\right|_{q}} \mathrm{~dB}$

and

$\left.\mathrm{SE}_{m}\right|_{q}=20 \log _{10} \frac{\left|\boldsymbol{H}^{u s}\right|_{q}}{\left|\boldsymbol{H}^{s}\right|_{q}} \mathrm{~dB}$

$\boldsymbol{E}^{u s}$ and $\boldsymbol{H}^{u s}$ are the phasors of the electric and the magnetic field intensities in the absence of the shield at the point $q$, while $\boldsymbol{E}^{s}$ and $\boldsymbol{H}^{s}$ are the phasors of the field intensities in the presence of the shield, at the same point $q$. Throughout the paper, phasors are defined with respect to a time factor $e^{+j \omega t}$.

To come to analytically treatable expressions, remember that any electromagnetic field in a homogeneous domain outside the sources can be described by a spherical multipole expansion (Stratton, 1941) as

$$
\begin{aligned}
\boldsymbol{E}(\boldsymbol{r}) & =\sum_{n=1}^{\infty} \sum_{m=-n}^{n}\left[A_{n m} \boldsymbol{N}_{n m}+\frac{Z}{j} B_{n m} \boldsymbol{M}_{n m}\right] \\
\boldsymbol{H}(\boldsymbol{r}) & =\sum_{n=1}^{\infty} \sum_{m=-n}^{n}\left[\frac{j}{Z} A_{n m} \boldsymbol{M}_{n m}+B_{n m} \boldsymbol{N}_{n m}\right]
\end{aligned}
$$

where $Z=\sqrt{\frac{\mu}{\epsilon}}$ denotes the intrinsic impedance of the medium, $A_{n m}$ and $B_{n m}$ are referred to as the electric and magnetic multipole amplitudes, and $\boldsymbol{M}_{n m}$ and $\boldsymbol{N}_{n m}$ are the solenoidal (divergence-free) expansion functions. The latter 
are defined by

$$
\begin{aligned}
\boldsymbol{M}_{n m}(\boldsymbol{r})= & z_{n}(\kappa r) \boldsymbol{m}_{n m}(\vartheta, \varphi) \\
\boldsymbol{N}_{n m}(\boldsymbol{r})= & -\frac{z_{n}(\kappa r)}{\kappa r} n(n+1) Y_{n m}(\vartheta, \varphi) \boldsymbol{e}_{r} \\
& -\frac{1}{\kappa r} \frac{d}{d r}\left\{r z_{n}(\kappa r)\right\} \boldsymbol{n}_{n m}(\vartheta, \varphi)
\end{aligned}
$$

where $\kappa=\omega \sqrt{\mu \epsilon}$ denotes the wave number and $r, \vartheta, \varphi$ are the coordinates of a spherical coordinate system. The relation between the spherical cylinder functions $z_{n}(\kappa r)$ and the cylinder functions $Z_{n}(\kappa r)$ is given by (Abramowitz and Stegun, 1972)

$z_{n}(\kappa r)=\sqrt{\frac{\pi}{2 \kappa r}} Z_{n+\frac{1}{2}}(\kappa r)$.

The transverse vector functions $\boldsymbol{m}_{n m}$ and $\boldsymbol{n}_{n m}$

$$
\begin{aligned}
\boldsymbol{m}_{n m}(\vartheta, \varphi)=-\frac{1}{\sin \vartheta} \frac{\partial}{\partial \varphi}\left\{Y_{n m}(\vartheta, \varphi)\right\} \boldsymbol{e}_{\vartheta} \\
+\frac{\partial}{\partial \vartheta}\left\{Y_{n m}(\vartheta, \varphi)\right\} \boldsymbol{e}_{\varphi}, \\
\boldsymbol{n}_{n m}(\vartheta, \varphi)=\quad \begin{aligned}
& \frac{\partial}{\partial \vartheta}\left\{Y_{n m}(\vartheta, \varphi)\right\} \boldsymbol{e}_{\vartheta} \\
&+\frac{1}{\sin \vartheta} \frac{\partial}{\partial \varphi}\left\{Y_{n m}(\vartheta, \varphi)\right\} \boldsymbol{e}_{\varphi}
\end{aligned}
\end{aligned}
$$

are related to the surface spherical harmonics by

$$
Y_{n m}(\vartheta, \varphi)=\sqrt{\frac{2 n+1}{4 \pi} \frac{(n-m) !}{(n+m) !}} P_{n}^{m}(\cos \vartheta) e^{j m \varphi}
$$

with $P_{n}^{m}(\cos \vartheta)$ being associated Legendre functions of the 1 st kind.

We choose the aforementioned point $q$ as the origin of the spherical coordinate system $\boldsymbol{r}$ and have thus - because of the regularity condition - to use spherical Bessel functions of the 1st kind, $j_{n}(\kappa r)$, as the spherical cylinder functions in (4), since we want to describe the fields at the origin. This fact will be indicated by an upper index ${ }^{I}$ as:

$$
\begin{aligned}
\boldsymbol{E}(\boldsymbol{r}) & =\sum_{n=1}^{\infty} \sum_{m=-n}^{n}\left[A_{n m} \boldsymbol{N}_{n m}^{I}(\boldsymbol{r})+\frac{Z}{j} B_{n m} \boldsymbol{M}_{n m}^{I}(\boldsymbol{r})\right] \\
\boldsymbol{H}(\boldsymbol{r}) & =\sum_{n=1}^{\infty} \sum_{m=-n}^{n}\left[\frac{j}{Z} A_{n m} \boldsymbol{M}_{n m}^{I}(\boldsymbol{r})+B_{n m} \boldsymbol{N}_{n m}^{I}(\boldsymbol{r})\right] .
\end{aligned}
$$

Here, the multipole amplitudes $\left(A_{n m}, B_{n m}\right)$ for an electric dipole located at $\boldsymbol{r}^{\prime}\left(r^{\prime}>0\right)$, described by the current density $\boldsymbol{J}(\boldsymbol{r})=\boldsymbol{c}_{e l} \delta\left(\boldsymbol{r}-\boldsymbol{r}^{\prime}\right)$ with a current moment $\boldsymbol{c}_{e l}$, can be obtained by evaluating the dyadic Green's function of the free space (Klinkenbusch, 1996) as

$$
\begin{aligned}
& A_{n m}=-\kappa^{2} Z \frac{(-1)^{m}}{n(n+1)} N_{n,-m}^{I I}\left(\boldsymbol{r}^{\prime}\right) \cdot \boldsymbol{c}_{e l} \\
& B_{n m}=-j \kappa^{2} \frac{(-1)^{m}}{n(n+1)} \boldsymbol{M}_{n,-m}^{I I}\left(\boldsymbol{r}^{\prime}\right) \cdot \boldsymbol{c}_{e l}
\end{aligned}
$$

where the upper index ${ }^{I I}$ stands for the use of spherical Hankel functions of the 2nd kind $h_{n}^{(2)}(\kappa r)$. As mentioned we will be interested in the fields in the immediate vicinity of the point $q(r=0)$, and thus will have a look at the behavior of the spherical Bessel function of the 1st kind for small values of its argument (Abramowitz and Stegun, 1972):

$$
\begin{aligned}
& \lim _{\kappa r \rightarrow 0} j_{n}(\kappa r)=0, \quad n \geq 1 \\
& \lim _{\kappa r \rightarrow 0} \frac{j_{n}(\kappa r)}{\kappa r}= \begin{cases}\frac{1}{3}, & \text { if } n=1 \\
0, & \text { if } n>1 \\
\frac{2}{3}, & \text { if } n=1 \\
0, & \text { if } n>1 .\end{cases} \\
& \lim _{\kappa r \rightarrow 0} \frac{1}{\kappa r} \frac{d}{d r}\left\{r j_{n}(\kappa r)\right\}
\end{aligned}
$$

Hence we need multipole fields of order $n=1$ only to get a complete field expansion at the point $q$. Inserting (10) in (4) leads to

$$
\begin{aligned}
& \left|N_{1, m}^{I}(\boldsymbol{r})\right|_{r=0}^{2}=\frac{1}{3 \pi} \quad \text { for } m \in\{-1,0,1\} . \\
& \left|\boldsymbol{M}_{1, m}^{I}(\boldsymbol{r})\right|_{r=0}^{2}=0
\end{aligned}
$$

We now expand both, the electromagnetic fields in the absence and in the presence of the shield (that is, in the unshielded and in the shielded case, respectively) in terms of spherical multipole expansions with $n=1$, and denote the multipole amplitudes by $\left(A_{n, m}^{u s}, B_{n, m}^{u s}\right)$ and $\left(A_{n, m}^{s}, B_{n, m}^{s}\right)$, respectively. Then we finally may write the electric and magnetic SE at the point $q$ as:

$\left.\mathrm{SE}_{e}\right|_{q}=20 \log _{10} \frac{\sum_{m=-1}^{1}\left|A_{1, m}^{u s}\right|}{\sum_{m=-1}^{1}\left|A_{1, m}^{s}\right|} \mathrm{dB}$

and

$\left.\mathrm{SE}_{m}\right|_{q}=20 \log _{10} \frac{\sum_{m=-1}^{1}\left|B_{1, m}^{u s}\right|}{\sum_{m=-1}^{1}\left|B_{1, m}^{s}\right|} \mathrm{dB}$.

Note that these definitions of the electric and magnetic SE are independent of the kind of the shield and of the incident field, in particular, they are valid for both far-field as well as for near-field sources.

\subsection{Transient Shielding Effectiveness}

We first introduce the relations between the Fourier transforms $(\mathcal{E}, \mathcal{H})$ and the phasors $(\boldsymbol{E}, \boldsymbol{H})$ of the field intensities at $q$ in the unshielded (free space) case as

$$
\begin{aligned}
\left.\mathcal{E}\right|_{q}(\omega) & =\left.S(\omega) \boldsymbol{E}\right|_{q} \\
\left.\mathcal{H}\right|_{q}(\omega) & =\left.S(\omega) \boldsymbol{H}\right|_{q}
\end{aligned}
$$

provided that the field sources are located in the far field. $S(\omega)$ is the spectral density distribution of the incident field and contains its complete frequency information at $q$. For instance, (14) can be achieved by an incident plane wave with 
a constant amplitude or by a Hertzian-dipole source (with an appropriately chosen current moment) located in the far field. Note that we shall investigate a Hertzian-dipole nearfield source in the following, and that we choose its current moment such that the field would satisfy (14) if it would be moved to the far field.

Consider now a small lossy sphere, concentrically located around the point $q$. This load is either shielded or nonshielded against an external transient electromagnetic field (i.e., an impulse). The ratio of the absorbed electromagnetic energies in the absence and the presence of the shield

$a_{W}=10 \log _{10} \frac{W^{u s}}{W^{s}}$

might be considered as an optimal quantity to characterize the shielding effectiveness in case of transient fields. For practical reasons, $a_{W}$ is not a suitable definition in general, however, as shown in (Klinkenbusch, 2005), in the limiting case of a vanishing load $a_{W}$ turns into the transient SE at the point $q$, which can be evaluated by

$$
\begin{aligned}
& \left.\mathrm{SE}_{t}\right|_{q}= \\
& 10 \log _{10} \frac{\int_{0}^{\infty}|S(\omega)|^{2} \sum_{m=-1}^{1}\left[\left|A_{1 m}^{u s}\right|^{2}+Z^{2}\left|B_{1 m}^{u s}\right|^{2}\right] \omega d \omega}{\int_{0}^{\infty}|S(\omega)|^{2} \sum_{m=-1}^{1}\left[\left|A_{1 m}^{s}\right|^{2}+Z^{2}\left|B_{1 m}^{s}\right|^{2}\right] \omega d \omega} \mathrm{dB} .
\end{aligned}
$$

Now consider an electric (Hertzian) dipole located at $\boldsymbol{r}=$ $\boldsymbol{r}^{\prime}$ outside a shielding structure. Assume that the dipole is polarized perpendicularly with respect to $\boldsymbol{r}^{\prime}$, and that the corresponding electric current moment may be given by $\boldsymbol{c}_{e l}=c_{e l} \boldsymbol{e}_{\vartheta}$. Note that $\omega c_{e l}$ is chosen to be independent of $\omega$ to ensure that the frequency dependence at $q$ is solely described by $S(\omega)$ if the Hertzian dipole is located in the far field. To evaluate (9), we will have a look at the behavior of the Hankel functions of the 2nd kind for $n=1$ :

$$
\begin{aligned}
h_{1}^{(2)}\left(\kappa r^{\prime}\right) & =\left(\frac{-1}{\kappa r^{\prime}}+\frac{j}{\left(\kappa r^{\prime}\right)^{2}}\right) e^{-j \kappa r^{\prime}} \\
\frac{1}{\kappa r^{\prime}} h_{1}^{(2)}\left(\kappa r^{\prime}\right) & =\left(\frac{-1}{\left(\kappa r^{\prime}\right)^{2}}+\frac{j}{\left(\kappa r^{\prime}\right)^{3}}\right) e^{-j \kappa r^{\prime}} \\
\frac{1}{\kappa r^{\prime}} \frac{\partial}{\partial r^{\prime}}\left\{r^{\prime} h_{1}^{(2)}\left(\kappa r^{\prime}\right)\right\} & =\left(\frac{j}{\kappa r^{\prime}}+\frac{1}{\left(\kappa r^{\prime}\right)^{2}}+\frac{-j}{(\kappa r)^{3}}\right) e^{-j \kappa r^{\prime}} .
\end{aligned}
$$

For the unshielded case, the multipole amplitudes can then be written as

$$
\begin{aligned}
& A_{1,1}^{u s}=-\frac{\kappa^{2} Z}{2} c_{e l} \sqrt{\frac{3}{8 \pi}}\left[\frac{-j}{\left(\kappa r^{\prime}\right)^{3}}+\frac{1}{\left(\kappa r^{\prime}\right)^{2}}+\frac{j}{\kappa r^{\prime}}\right] e^{-j \varphi} e^{-j \kappa r^{\prime}} \\
& A_{1,0}^{u s}=0 \\
& A_{1,-1}^{u s}=\frac{\kappa^{2} Z}{2} c_{e l} \sqrt{\frac{3}{8 \pi}}\left[\frac{-j}{\left(\kappa r^{\prime}\right)^{3}}+\frac{1}{\left(\kappa r^{\prime}\right)^{2}}+\frac{j}{\kappa r^{\prime}}\right] e^{j \varphi} e^{-j \kappa r^{\prime}} \\
& B_{1,1}^{u s}=\frac{\kappa^{2}}{2} c_{e l} \sqrt{\frac{3}{8 \pi}}\left[\frac{-j}{\left(\kappa r^{\prime}\right)^{2}}+\frac{1}{\kappa r^{\prime}}\right] e^{-j \varphi} e^{-j \kappa r^{\prime}} \\
& B_{1,0}^{u s}=0 \\
& B_{1,1}^{u s}=\frac{\kappa^{2}}{2} c_{e l} \sqrt{\frac{3}{8 \pi}}\left[\frac{-j}{\left(\kappa r^{\prime}\right)^{2}}+\frac{1}{\kappa r^{\prime}}\right] e^{j \varphi} e^{-j \kappa r^{\prime}}
\end{aligned}
$$

Introducing the factors

$$
\begin{aligned}
& g=\kappa \sqrt{\frac{1}{\left(\kappa r^{\prime}\right)^{6}}-\frac{1}{\left(\kappa r^{\prime}\right)^{4}}+\frac{1}{\left(\kappa r^{\prime}\right)^{2}}} \\
& h=\kappa \sqrt{\frac{1}{\left(\kappa r^{\prime}\right)^{4}}+\frac{1}{\left(\kappa r^{\prime}\right)^{2}}}
\end{aligned}
$$

we can derive relations between the magnitudes of the multipole amplitudes for the unshielded case as

$$
\frac{\left|A_{1,1}^{u s}\right|}{g}=\frac{\left|A_{1,-1}^{u s}\right|}{g}=Z \frac{\left|B_{1,1}^{u s}\right|}{h}=Z \frac{\left|B_{1,-1}^{u s}\right|}{h} .
$$

The quantities in (20) do not depend on the frequency, and we can finally re-write the transient SE in (16) for a perpendicularly polarized electric dipole as

$$
\left.\mathrm{SE}_{t}\right|_{q}=10 \log _{10} \frac{\int_{0}^{\infty}|S(\omega)|^{2}\left[g^{2}+h^{2}\right] \omega d \omega}{\int_{0}^{\infty}|S(\omega)|^{2}\left[g^{2} \frac{\left|\boldsymbol{E}^{s}\right|_{q}^{2}}{\left|\boldsymbol{E}^{u s}\right|_{q}^{2}}+h^{2} \frac{\left|\boldsymbol{H}^{s}\right|_{q}^{2}}{\left|\boldsymbol{H}^{u s}\right|_{q}^{2}}\right] \omega d \omega} \mathrm{dB} .
$$

Note that the expression (21) implies a simple measurement procedure, as in the case of an incident plane wave.

\subsection{Electromagnetic Shielding Effectiveness}

The electromagnetic SE can be seen as a special case of the transient SE. We simply need the spectral density distribution $S(\omega)$ of a time harmonic source at circle frequency $\omega_{0}$, which essentially is given by the Dirac distribution $\delta\left(\omega-\omega_{0}\right)$. To now derive the electromagnetic SE, we first introduce (for the sake of clarity) the functions

$$
\begin{aligned}
& f_{1}(\omega)=\sum_{m=-1}^{1}\left[\left|A_{1 m}^{u s}\right|^{2}+Z^{2}\left|B_{1 m}^{u s}\right|^{2}\right] \omega \\
& f_{2}(\omega)=\sum_{m=-1}^{1}\left[\left|A_{1 m}^{s}\right|^{2}+Z^{2}\left|B_{1 m}^{s}\right|^{2}\right] \omega .
\end{aligned}
$$

We have to evaluate $|S(\omega)|^{2}$, however, because $\left|\delta\left(\omega-\omega_{0}\right)\right|^{2}$ is not defined, we use the $\delta$-convergent series:

$\delta\left(\omega-\omega_{0}\right)=\lim _{\Delta \omega \rightarrow 0} \frac{1}{\Delta \omega} \operatorname{rect}\left(\frac{\omega-\omega_{0}}{\Delta \omega}\right)$.

With (22) and (23) the electromagnetic SE (16) for a timeharmonic process can be written as

$$
\left.\mathrm{SE}_{e m}\right|_{q}=10 \log _{10} \frac{\int_{0}^{\infty} \lim _{\Delta \omega \rightarrow 0}\left(\frac{1}{\Delta \omega}\right)^{2} \operatorname{rect}^{2}\left(\frac{\omega-\omega_{0}}{\Delta \omega}\right) f_{1}(\omega) d \omega}{\int_{0}^{\infty} \lim _{\Delta \omega \rightarrow 0}\left(\frac{1}{\Delta \omega}\right)^{2} \operatorname{rect}^{2}\left(\frac{\omega-\omega_{0}}{\Delta \omega}\right) f_{2}(\omega) d \omega} \mathrm{dB}
$$




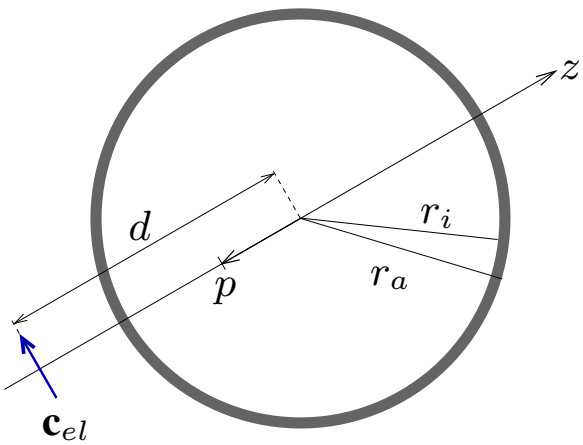

Fig. 1. Spherical shell $\left(r_{i}=0.5 \mathrm{~m}, r_{a}=0.51 \mathrm{~m}\right)$, and locations of the measurement point $z=p$ and the Hertzian dipole at $z=-d$ $\left(d>r_{a}\right)$.

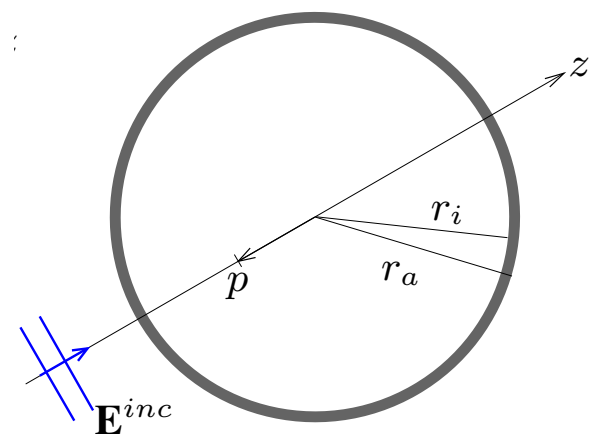

Fig. 2. Spherical shell $\left(r_{i}=0.5 \mathrm{~m}, r_{a}=0.51 \mathrm{~m}\right)$, and location of the measurement point at $z=p$ for an incident plane wave.

which is because of $\operatorname{rect}^{2}(X)=\operatorname{rect}(X)$

$$
\begin{aligned}
\left.\mathrm{SE}_{e m}\right|_{q} & =10 \log _{10} \lim _{\Delta \omega \rightarrow 0} \frac{\int_{0}^{\infty}\left(\frac{1}{\Delta \omega}\right)^{2} \operatorname{rect}\left(\frac{\omega-\omega_{0}}{\Delta \omega}\right) f_{1}(\omega) d \omega}{\int_{0}^{\infty}\left(\frac{1}{\Delta \omega}\right)^{2} \operatorname{rect}\left(\frac{\omega-\omega_{0}}{\Delta \omega}\right) f_{2}(\omega) d \omega} \mathrm{dB} \\
& =10 \log _{10} \frac{\int_{0}^{\infty} \delta\left(\omega-\omega_{0}\right) f_{1}(\omega) d \omega}{\int_{0}^{\infty} \delta\left(\omega-\omega_{0}\right) f_{2}(\omega) d \omega} d B \\
& =10 \log _{10} \frac{f_{1}\left(\omega_{0}\right)}{f_{2}\left(\omega_{0}\right)} \mathrm{dB}
\end{aligned}
$$

With (22) we obtain for the electromagnetic SE

$\left.\mathrm{SE}_{e m}\right|_{q}=10 \log _{10} \frac{\sum_{m=-1}^{1}\left[\left|A_{1 m}^{u s}\right|^{2}+Z^{2}\left|B_{1 m}^{u s}\right|^{2}\right]}{\sum_{m=-1}^{1}\left[\left|A_{1 m}^{s}\right|^{2}+Z^{2}\left|B_{1 m}^{s}\right|^{2}\right]} \mathrm{dB}$.

Similarly to the derivation in the transient case we find the electromagnetic SE for an electric dipole located at $\boldsymbol{r}^{\prime}$ and polarized perpendicularly to $\boldsymbol{r}^{\prime}$ by inserting (20) into (26).

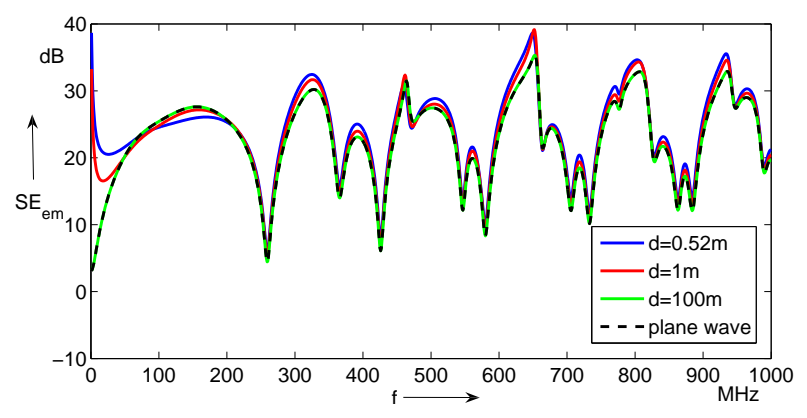

Fig. 3. $\mathrm{SE}_{e m}$ at $q=-0.1 \mathrm{~m}\left(\sigma=10 \frac{\mathrm{S}}{\mathrm{m}}\right)$ for different dipole distances and for an incident plane wave.

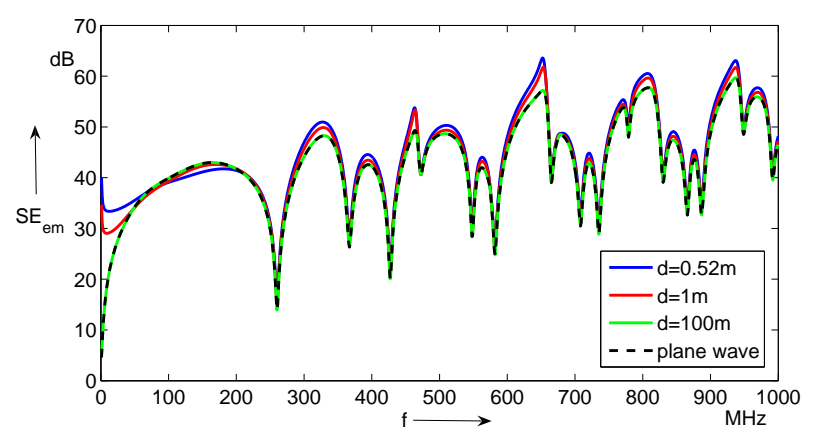

Fig. 4. $\mathrm{SE}_{e m}$ at $p=-0.1 \mathrm{~m}\left(\sigma=50 \frac{\mathrm{S}}{\mathrm{m}}\right)$ for different dipole distances and for an incident plane wave.

With (1), (2), (12), and (13) we finally are led to

$\left.\mathrm{SE}_{e m}\right|_{q}=10 \log _{10} \frac{g^{2}+h^{2}}{g^{2} \frac{\left|\boldsymbol{E}^{s}\right|_{q}^{2}}{\left|\boldsymbol{E}^{u s}\right|_{q}^{2}}+h^{2} \frac{\left|\boldsymbol{H}^{s}\right|_{q}^{2}}{\left|\boldsymbol{H}^{u s}\right|_{q}^{2}}} \mathrm{~dB}$.

2.4 Asymptotic behavior if the dipole is located in the far field

If the perpendicularly polarized electric dipole is located in the far field of the shield $\left(\kappa r^{\prime} \gg 1\right)$ we expect results for both the transient and the electromagnetic SE which we have obtained for an incident plane wave. To show that, we first will have to look at the factors $g$ and $h$ in (19) for this case, and we easily see that it holds

$g \approx \frac{1}{r^{\prime}} ; h \approx \frac{1}{r^{\prime}} \quad\left(\right.$ if $\left.\kappa r^{\prime} \gg 1\right)$.

It immediately follows that (21) turns into the transient SE for an incident plane wave

$$
\left.\mathrm{SE}_{t}\right|_{q}=10 \log _{10} \frac{2 \int_{0}^{\infty}|S(\omega)|^{2} \omega d \omega}{\int_{0}^{\infty}|S(\omega)|^{2}\left[\frac{\left|\boldsymbol{E}^{s}\right|_{q}^{2}}{\left|\boldsymbol{E}^{u s}\right|_{q}^{2}}+\frac{\left|\boldsymbol{H}^{s}\right|_{q}^{2}}{\left|\boldsymbol{H}^{u s}\right|_{q}^{2}}\right] \omega d \omega} \mathrm{dB}
$$




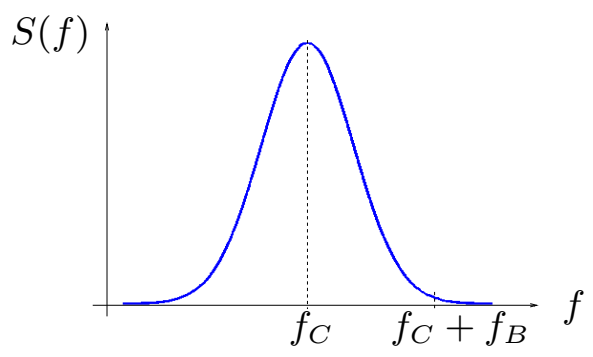

Fig. 5. Gaussian spectral density distribution

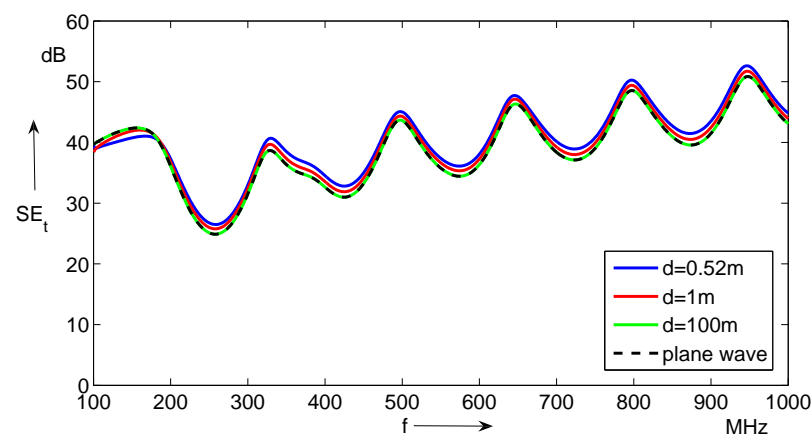

Fig. 6. $\mathrm{SE}_{t}$ at $p=-0.1 \mathrm{~m}\left(\sigma=50 \frac{\mathrm{S}}{\mathrm{m}}\right)$ for different dipole distances and for an incident plane wave, as a function of the center-frequency of the Gaussian-type impulse with bandwidth $f_{B}=100 \mathrm{MHz}$.

and that (27) turns into the electromagnetic SE for an incident plane wave:

$\left.\mathrm{SE}_{e m}\right|_{q}=10 \log _{10} \frac{2}{\frac{\left|\boldsymbol{E}^{s}\right|_{q}^{2}}{\left|\boldsymbol{E}^{u s}\right|_{q}^{2}}+\frac{\left|\boldsymbol{H}_{q}^{s}\right|^{2}}{\left|\boldsymbol{H}_{q}^{u s}\right|^{2}}} \mathrm{~dB}$.

\section{Numerical results}

The new expressions for the transient and the electromagnetic SE for an electric-dipole near-field source have been numerically evaluated for a shielding structure which consists of a closed spherical shell.

In each of the following cases, the SE is determined at the point $z=p$, which is inside the spherical shell on the $z$-axis of a coordinate system with origin at the shell's center. The Hertzian dipole lies on the negative $z$-axis at $z=-d\left(d>r_{a}\right.$, see Fig. 1), moreover the plane wave (We shall need this case for validating some results.) is incident from the negative $z$ axis (see Fig. 2).

In all evaluations the inner and outer radius of the spherical shell are chosen as $r_{i}=0.5 \mathrm{~m}$ and $r_{a}=0.51 \mathrm{~m}$, respectively. At first, Figures 3 and 4 show the electromagnetic $\mathrm{SE}$ at $p=-0.1 \mathrm{~m}$ for 2 different values of the shield conduc-

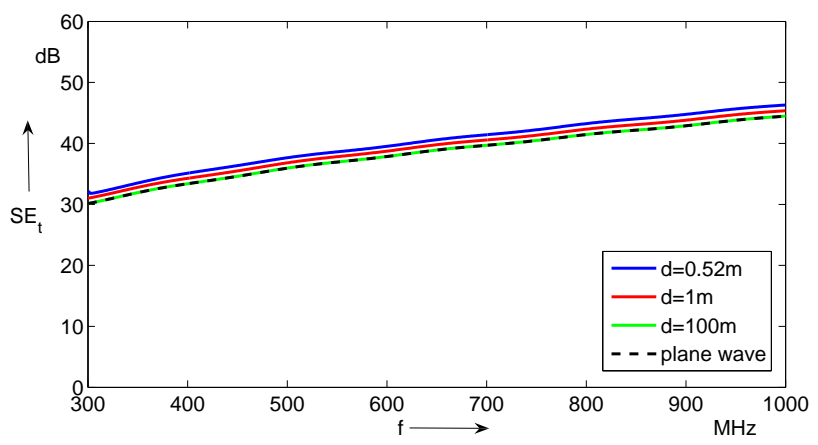

Fig. 7. $\mathrm{SE}_{t}$ at $p=-0.1 \mathrm{~m}\left(\sigma=50 \frac{\mathrm{S}}{\mathrm{m}}\right)$ for different dipole distances and for an incident plane wave, as a function of the center-frequency of the Gaussian-type impulse with bandwidth $f_{B}=300 \mathrm{MHz}$.

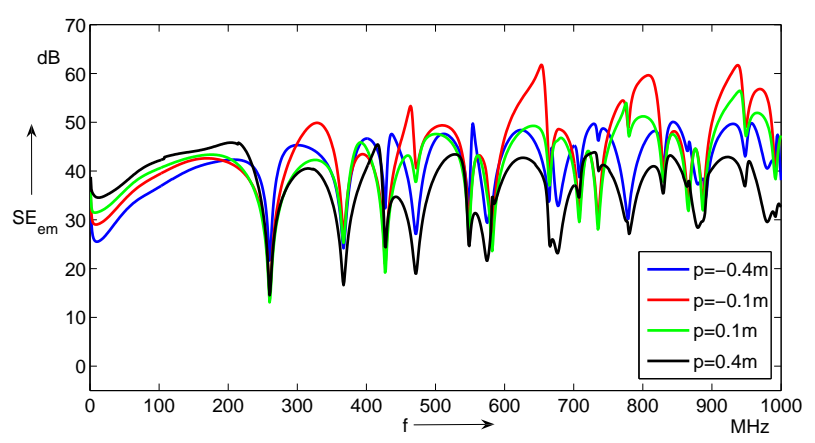

Fig. 8. $\mathrm{SE}_{\text {em }}$ at different measurement locations $z=p$ for a Hertzian-dipole source located at $d=1 \mathrm{~m}\left(\sigma=50 \frac{\mathrm{S}}{\mathrm{m}}\right)$.

tivity, $\sigma=10 \frac{\mathrm{S}}{\mathrm{m}}$ and $\sigma=50 \frac{\mathrm{S}}{\mathrm{m}}$, respectively, each for different dipole positions as well as for an incident plane wave.

As expected the values of the SE obtained for dipoles located in the far field are identical to the values obtained for an incident plane wave. For smaller distances between dipole and shield, differences between the SE can be observed especially in the lower frequency range and at most of the SE maxima.

For the evaluation of the transient SE, in the following a Gaussian impulse with a spectral density distribution $S(f)$ as shown in Fig. 5 will be used, with the center frequency at $f=f_{C}$ and the bandwidth $f_{B}$ defined by:

$S\left(f_{C} \pm f_{B}\right)=\frac{1}{100} S\left(f_{C}\right)$.

Figures 6 and 7 represent the transient SE for different bandwidths as a function of the center frequency. Figures 6 and 7 show the transient SE for bandwidths $f_{B}=100 \mathrm{MHz}$ and $f_{B}=300 \mathrm{MHz}$, respectively.

For a higher bandwidth the curves of the transient SE become smoother, and for large distances of the Hertziandipole source, again the same transient SE as for an incident 


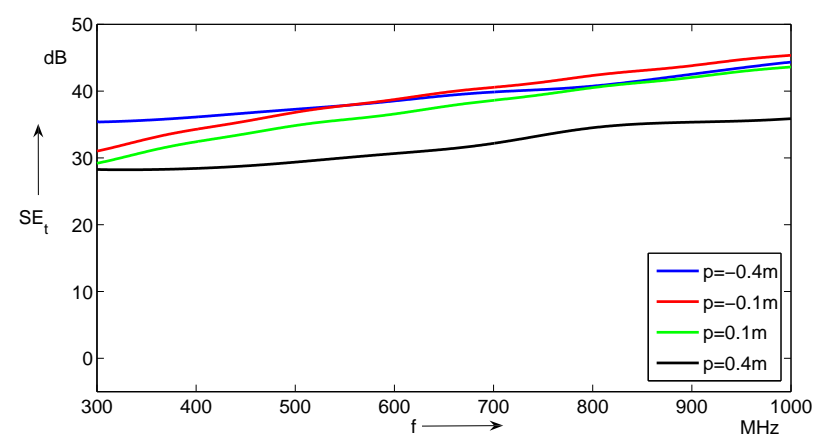

Fig. 9. $\mathrm{SE}_{t}$ at different measurement locations $z=p$ for a Hertziandipole source located at $d=1 \mathrm{~m}\left(\sigma=50 \frac{\mathrm{S}}{\mathrm{m}}, f_{B}=300 \mathrm{MHz}\right)$.

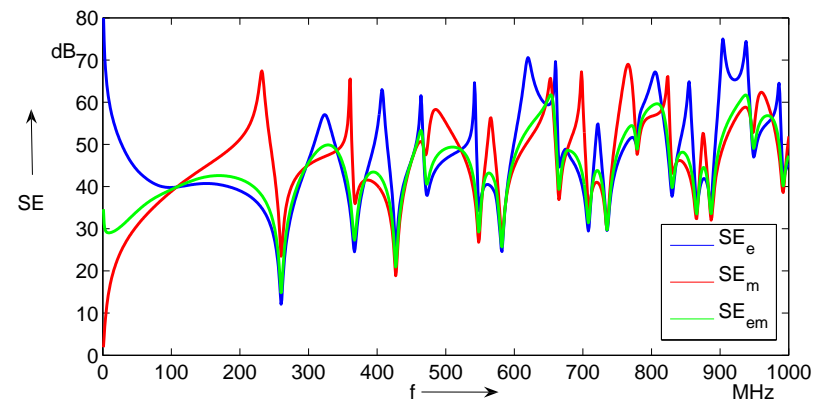

Fig. 10. $\mathrm{SE}_{e}, \mathrm{SE}_{m}$, and $\mathrm{SE}_{e m}$ at $p=-0.1 \mathrm{~m}$ for a Hertzian-dipole source at $d=1 \mathrm{~m}\left(\sigma=50 \frac{\mathrm{S}}{\mathrm{m}}\right)$.

plane wave is achieved. Figure 8 shows the electromagnetic $\mathrm{SE}$ at four different positions on the $z$-axis inside the spherical shell in case of an electric-dipole near-field source located at $d=1 \mathrm{~m}$. The curves are different in amplitude though they show a similar frequency dependence. The corresponding transient SE for a Gaussian impulse with a bandwidth $f_{B}=300 \mathrm{MHz}$ is plotted in Fig. 9 .

Finally, Fig. 10 shows the electric, magnetic and electromagnetic shielding effectiveness at $a=-0.1 \mathrm{~m}$ for a Hertzian-dipole source located at $d=1 \mathrm{~m}$. As in case of an incident plane wave the value of the electromagnetic SE always lies between the values of the electric and magnetic SE.

\section{Conclusions}

We have investigated the electromagnetic and transient shielding effectiveness (SE) for an electric dipole located in the near field of the shield as the source. It has been shown both analytically and numerically, that for large distances between the dipole source and the shield the results exactly turn into the values which we obtain with the plane-wave definitions. From the numerical results, which have been exemplarily performed for a closed spherical shell as the shielding structure, it turned out that the proposed SE definitions are useful and valid in case of near-field sources as well. As the SE values depend on the measurement position inside the shield, in practice one will have to ensure that the SE measurements are done at several and/or typical measurement points. Moreover, it has to be emphasized that the main content of any SE information is to compare the shielding abilities of two or more comparable shielding structures.

\section{References}

Abramowitz, M. and Stegun, I.: Handbook of Mathematical Functions with Formulas, Graphs, and Mathematical Tables, Department of Commerce - United States of America, Washington, 10th gpo printing edn., 1972.

Kaden, H.: Wirbelströme und Schirmung in der Nachrichtentechnik, Springer, Berlin, Göttingen, Heidelberg, 2nd edn., 1959.

Klinkenbusch, L.: Theorie der sphärischen Absorberkammer und des mehrschaligen Kugelschirmes - Multipolanalyse des elektromagnetischen Feldes in sphärisch geschichteten Strukturen, Ruhr-Universtität Bochum, 1996.

Klinkenbusch, L.: On the Shielding Effectiveness of Enclosures, IEEE Transactions on Electromagnetic Compatibility, 47, 589601, 2005.

Stratton, J.: Electromagnetic Theory, McGraw-Hill Book Company Inc., New York, 1941. 